\title{
Role of VEGFA gene polymorphisms in colorectal cancer patients who treated with bevacizumab
}

\author{
Wei Cui ${ }^{1}$, Feng Li ${ }^{2}$, Qiang Yuan ${ }^{1}$, Gang Chen ${ }^{1}$, Cailing Chen ${ }^{1}$ and Bo Yu ${ }^{1}$ \\ ${ }^{1}$ Department of General Surgery, The Military General Hospital of Beijing PLA, Beijing 100700, China \\ ${ }^{2}$ Department of Health, The Military General Hospital of Beijing PLA, Beijing 100700, China \\ Correspondence to: Bo YU, email: sdklfod@yeah.net \\ Keywords: VEGFA; polymorphisms; bevacizumab; colorectal cancer \\ Received: March 21, $2017 \quad$ Accepted: August 26, $2017 \quad$ Published: November 06, 2017 \\ Copyright: Cui et al. This is an open-access article distributed under the terms of the Creative Commons Attribution License 3.0 \\ (CC BY 3.0), which permits unrestricted use, distribution, and reproduction in any medium, provided the original author and source \\ are credited.
}

\section{ABSTRACT}

Objectives: This study aimed to explore the effects of vascular endothelial growth factor A (VEGFA) gene polymorphisms (rs699947 and rs833061) on Bevacizumab (BEV) treatment in colorectal cancer (CRC) patients.

Methods: 125 CRC cases receiving BEV plus FOLFIRI treatment were recruited in this study. VEGFA polymorphisms were genotyped using polymerase chain reactionrestriction fragment length polymorphism (PCR-RFLP) method. Correlation of VEGFA gene polymorphisms with the response rate and progression free survival (PFS) was evaluated. Multivariate analyses were performed to estimate the effects of VEGFA polymorphisms on the therapeutic effects of BEV treatment in CRC patients.

Results: Rs699947 variants did not show significant association with BEV treatment. For rs833061 analysis, TT and TC genotype carriers had significantly higher ORR (objective response rate) than CC carriers $(P=0.048$ and $P=0.021$, respectively). Moreover, TT carriers underwent a well DCR (disease control rate) compared to CC carriers $(P=0.002)$. PFS time also showed obvious correlation with rs833061 polymorphism (log rank test, $P=\mathbf{0 . 0 0 2}$ ). Multivariate analyses demonstrated that TT and TC genotypes of rs833061 polymorphism were significantly correlated with enhanced therapeutic effects and prolonged PFS in CRC patients.

Conclusion: VEGFA rs833061 polymorphism is significantly associated with the therapeutic efficiency of bevacizumab in CRC patients.

\section{INTRODUCTION}

Colorectal cancer (CRC) is one of the most common malignancies, which is caused by the abnormal growth of cells in colon or rectum part. The tumor cell could spread elsewhere in the body. Recent years, with the development of therapy method, the mortality rate of CRC exhibits decreased trend [1]. CRC is caused by various genetic and environmental factors [2-5]. In the tumor growth and metastasis, angiogenesis is an essential factor [6]. Vascular endothelial growth factor (VEGF), a super family of growth factors, is involved in both vasculogenesis and angiogenesis $[7,8]$. It has been reported that the inhibition of angiogenesis will contribute to anti-tumor treatments
$[9,10]$. Recent decades, many angiogenesis inhibitors have been applied for cancer therapy. Bevacizumab (BEV) is a humanized monoclonal antibody which could suppress the angiogenesis by inhibiting VEGF binding to the VEGF receptors (VEGFR). Therefore, BEV regulates the angiogenesis via the inhibition of signal transduction. $\mathrm{BEV}$ has been used in the treatment of metastatic CRC. However, there was few clinical or biological factors to predict the therapeutic efficiency of BEV.

VEGFA is an important regulator of angiogensis in VEGF family, represents the main target of BEV. VEGFA plays a potential role in the development and treatment of CRC. Previous studies showed that polymorphisms of the $V E G F A$ gene influenced the expression or the function of 
its coded protein [11-13]. Therefore, the polymorphisms may have impact on the onset and progression of tumors $[14,15]$. It has been reported that single nucleotide polymorphisms (SNPs) of VEGFA gene could affect the tumor susceptibility, severity, and/or survival [16-18].

The role of VEGFA SNPs on the therapeutic efficiency of BEV-based patients has been investigated in many tumors [18-21]. For examples, Sohn et al. reported that TT genotype of VEGFA rs833061 polymorphism predicted superior ORR (objective response rate) to the first-line cytotoxic chemotherapy combined with bevacizumab among CRC patients in South Korea [21]. The GeparQuinto Phase III trial (NCT00567554) demonstrated that VEGFA gene rs699947 SNP was strongly correlated with pathological complete response among breast cancer patients receiving BEV-based neoadjuvant chemotherapy [22]. Based on the related investigations, we speculated that VEGFA gene rs699947 and rs833061 polymorphisms might influence treatment response among $\mathrm{CRC}$ patients treated by $\mathrm{BEV}$-based chemotherapy. However, there were merely no similar researches on the issue among Chinese Han CRC patients. Minor allele frequencies (MAF) of VEGFA gene rs699947 (c.-2578A $>$ C) and rs833061 (c.-1498C > T) polymorphisms are more than 0.05 in CHB (Chinese Han in Beijing) population. Therefore, we investigated the predictive function of VEGFA gene rs699947 and rs833061 polymorphisms in the outcomes of BEV-based therapy in Chinese Han CRC patients.

\section{RESULTS}

\section{Baseline characteristics}

A total of 125 (mean age 62.50 \pm 8.45 years) CRC patients receiving $\mathrm{BEV}$ combined FOLFIRI treatment were collected in this study Baseline characteristics of the participants were summarized in Table 1.

\section{Treatment response}

After three months treatment, 5 patients reached CR, 61 cases reached PR, 38 patients reached SD, and 21 cases presented PD. The ORR was $52.8 \%$, and DCR was $83.2 \%$. Meanwhile, the mean PFS were $19.67 \pm 0.919$ months during 30-month follow up investigation.

\section{Genotype distributions of $V E G F A$ rs699947 and rs833061 polymorphisms}

In the current study, PCR-RFLP was performed to identify the genotype of VEGFA rs699947 and rs 833061 polymorphisms. In addition, the results of 50 random samples were verified by direct sequencing. The genotype confirmed by PCR-RFLP and direct sequencing technology exhibited similar, suggesting the high accuracy of PCR-RFLP method in genotyping.
Genotypes and alleles distributions of rs699947 and rs833061 polymorphisms did not deviate from HWE test, showing the good representativeness of the objects. The genotype distributions of rs699947 and rs833061 among the study population were summarized in Table 2.

\section{Effects of rs699947 and rs833061 SNPs on BEV therapeutic efficiency}

In order to estimate the effects of rs699947 and rs833061 SNPs on BEV therapeutic efficiency, we compared ORR, DCR and PFS based on the genotypes of rs699947 and rs833061 polymorphisms (Table 2, Figure $1)$. In this study, there were no significant association observed between rs699947 polymorphism and ORR, DCR or PFS $(P>0.05$ for all, Table 2$)$. For rs833061 analysis, TT and TC genotype carriers had significantly higher ORR than CC carriers $(P=0.048$ and $P=0.021$, respectively). Moreover, TT carriers underwent a well DCR compared to $\mathrm{CC}$ carriers $(P=0.002)$. The PFS time also showed obvious correlation with rs833061 polymorphism (log rank test, $P=0.002$; significant level was adjusted by Bonferroni method, $\alpha=0.017$ ). TT carriers had a significantly better PFS than CC carriers (log rank test, $P=0.001$ ), while patients carrying TC genotype had obviously better PFS than those carrying CC genotype (log rank test, $P=0.013$ ). There was no remarkable difference between TT carriers and TC carriers (log rank test, $P=0.126$ ) (Figure 1).

After adjusted for age and gender, rs833061 TC carriers exhibited significantly higher ORR than CC carriers $(\mathrm{OR}=0.159,95 \% \mathrm{CI}=0.029-0.871, P=0.034)$, while TT carriers had a better DCR $(\mathrm{OR}=0.129,95 \% \mathrm{CI}=0.031$ 0.532, $P=0.002$ ) (Table 3). Univariate cox analysis demonstrated that TT and TC genotypes were protective factors for PFS ( $P=0.002$ and $P=0.048$, respectively). After adjusting to age and gender, TT $(\mathrm{HR}=0.299$, $95 \% \mathrm{CI}=0.141-0.634, P=0.002)$ and $\mathrm{TC} \quad(\mathrm{HR}=0.449$, $95 \% \mathrm{CI}=0.203-0.991, P=0.048)$ genotypes also showed obvious correlation with PFS (Table 3 ).

\section{DISCUSSION}

Accumulation of VEGF plays promoting roles in the development of tumors via enhancing angiogenesis. Among the VEGF family, VEGFA has the strongest activity to promote the angiogenesis. $\mathrm{BEV}$ is aimed at VEGF, weakens or blocks the interaction between VEGF and VEGFR, thus inhibits the proliferation of endothelial cell, reduces the angiogenesis in tumorigenesis. Inhibition of angiogenesis could prevent the growth of tumors and increase the therapeutic efficacy of radiotherapy and chemotherapy [23]. As one of the most common malignancy in large intestine, $\mathrm{CRC}$ has a high incidence and mortality. Recent years, relevant researches showed that therapeutic efficacy of BEV monotherapy is very 
Table 1: Baseline characteristics

\begin{tabular}{lc}
\hline Characteristics & Case number \\
\hline Age & \\
$\leq 60$ & $44(35.20)$ \\
$>60$ & $81(64.8)$ \\
Gender & \\
Male & $81(64.80)$ \\
Female & $44(35.20)$ \\
Primary tumor site & \\
Right colon & $33(26.40)$ \\
Left colon & $55(44.00)$ \\
Rectum & $37(29.60)$ \\
ECOG PS & \\
0 & $96(76.80)$ \\
$1-2$ & $29(23.20)$ \\
Primary tumor resected & \\
No & \\
Yes & $94(75.20)$ \\
Metastatic number & $31(24.80)$ \\
1 & \\
$>1$ & $58(46.40)$ \\
\hline
\end{tabular}

low [24], but the combination of chemotherapy and BEV significantly improve the therapeutic efficacy of advanced CRC [25]. However, the therapy response is different in the participants who receive the same therapy method. Additionally, the toxic and adverse effects are also different in the patients. These phenomena may be decided by the individual difference.

$V E G F A$, one of the target gene of $\mathrm{BEV}$, is located at $6 \mathrm{p} 12$, and contains 9 exons. It has been reported that polymorphisms in VEGFA gene play a potential role in the development of many tumors, including CRC [14, $15,26]$. Polymorphisms in this gene may impact the therapeutic efficiency of BEV. Ferroni et al demonstrated that -1154AA genotype was negatively correlated with efficacy and toxicity of bevacizumab in metastatic cancer patients [18]. Di and colleagues suggested that rs2010963 genotype was correlated with longer PFS in recurrent glioblastomas patients for the response to bevacizumab [20]. Rs699947 (c.-2578A>C) and rs833061 (c.-1498C>T) are the two widely researched SNPs in the promoter region of VEGFA gene. Previous studies showed that these polymorphisms could affect the promoter activity of the VEGFA gene, and then alter the production of VEGFA $[12,13]$. As a target of BEV treatment, abnormal accumulation of VAGFA might contribute to angiogenesis, thus leading to therapeutic failures. It was reported that high level of VEGFA predicted shorter recurrence-free survival among patients receiving bevacizumab-based chemotherapy [27]. Thus, polymorphisms at promoter region of VAGFA gene may significantly influence the therapeutic efficacy of BEV chemotherapy. Because the BEV monotherapy has low therapeutic efficacy, we used the combination chemotherapy to explore the effects of $V E G F A$ polymorphisms in the BEV therapeutic efficacy for CRC.

Growing evidences have suggested that BEV could significantly enhance the PFS of CRC patients who are treated with combination chemotherapy [25]. However, the therapeutic efficacy of BEV may exhibit individual differences. Genetic factors play pivotal roles in individual differences. To explore the association between SNPs and BEV treatments can help the physicians to identify the population who will benefit from BEV combined chemotherapy among advanced CRC patients, thus optimizing the management of BEV. In this study, we explored the association of $V E G F A$ polymorphisms with therapeutic efficacy of BEV in CRC patients. The results were estimated by ORR, DCR, and PFS. Analysis 
Table 2: Association between VEGFA gene polymorphisms and therapeutic effects of bevacizumab among CRC patients

\begin{tabular}{|c|c|c|c|c|c|c|c|}
\hline \multirow{2}{*}{ SNPs } & \multirow{2}{*}{ Total cases } & \multicolumn{2}{|c|}{ ORR } & \multicolumn{2}{|c|}{ DCR } & \multicolumn{2}{|l|}{ PFS } \\
\hline & & Case number & $P$ & Case number & $P$ & Mean time (months) & $P^{a}$ \\
\hline \multicolumn{8}{|c|}{ rs699947 } \\
\hline $\mathrm{CC}$ & $74(59.20)$ & $35(58.33)$ & 0.752 & $66(63.46)$ & 0.057 & $19.68 \pm 1.11$ & $\begin{array}{c}\log \text { rank test, } \\
P=0.392\end{array}$ \\
\hline $\mathrm{CA}$ & $46(36.80)$ & $29(48.33)$ & 0.316 & $35(33.65)$ & 0.433 & $20.98 \pm 1.73$ & \\
\hline AA & $5(4.00)$ & $2(3.33)$ & Reference & $3(2.88)$ & Reference & $16.60 \pm 4.63$ & \\
\hline \multicolumn{8}{|c|}{ rs833061 } \\
\hline TT & $79(63.20)$ & $42(63.64)$ & 0.048 & $70(67.31)$ & 0.002 & $20.41 \pm 1.20$ & $\begin{array}{c}\log \text { rank test, } \\
P=0.002^{*}\end{array}$ \\
\hline $\mathrm{TC}$ & $36(28.80)$ & $22(33.33)$ & 0.021 & $29(27.88)$ & 0.052 & $17.81 \pm 1.29$ & \\
\hline $\mathrm{CC}$ & $10(8.00)$ & $2(3.03)$ & Reference & $5(4.81)$ & Reference & $11.82 \pm 2.29$ & \\
\hline
\end{tabular}

Note: ${ }^{a}$ : significant level was adjusted by Bonferroni method, $\alpha=0.017$.

results indicated that rs699947 polymorphism did not show significant association with BEV treatment in CRC patients. Additionally, we found that the genotypes of the rs833061 SNP were significantly correlated with ORR, DCR and PFS among the study population. Multivariate analysis indicated that TC carriers had a higher ORR,

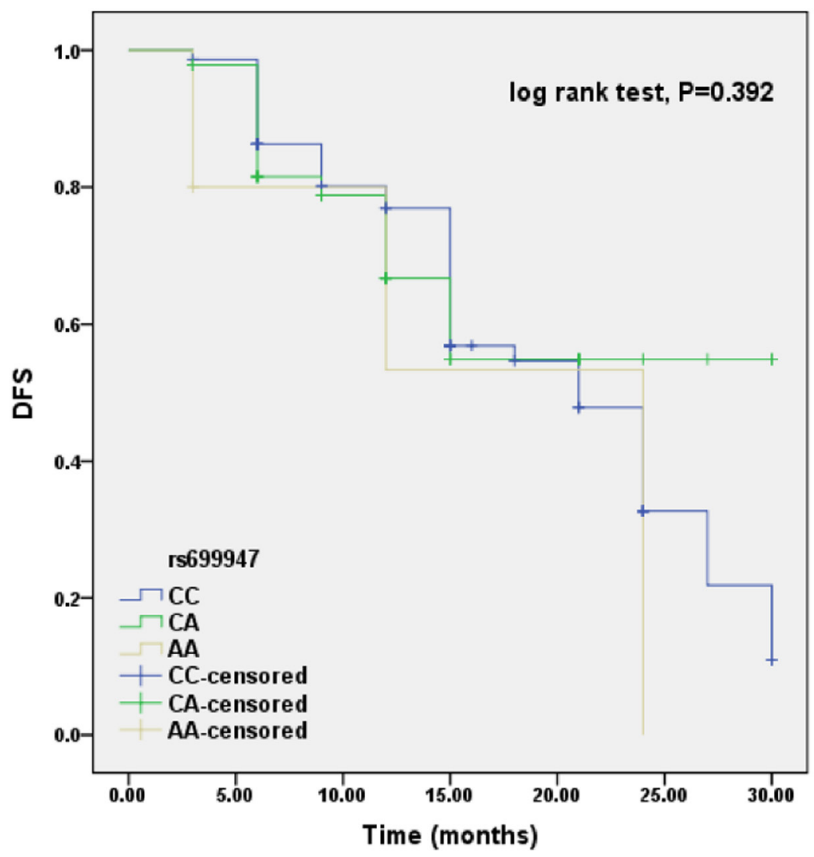

while TT carriers underwent well DCR. Furthermore, both of TC and TT carriers had a significantly better PFS than $\mathrm{CC}$ carriers. That was partly accordance with previous studies. Pallaud et al. found that among non-squamous non-small-cell lung cancer patients receiving BEV plus chemotherapy, TT carriers of rs833061 had the highest

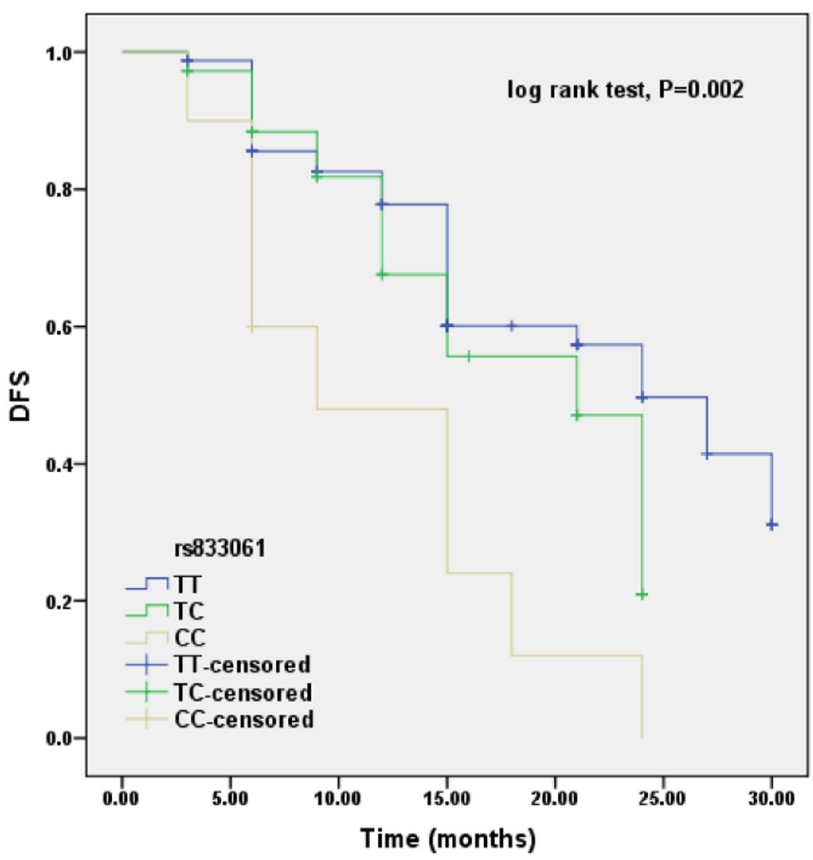

Figure 1: Effects of rs699947 and rs833061 polymorphisms on PFS among CRC patients receiving BEV treatments. There was no significant association between rs699947 polymorphism with PFC (log rank test, $P=0.392$ ). While rs 833061 polymorphism showed obvious correlation with PFS among the study population (log rank test, $P=0.002$ ). TT carriers had a significantly better PFS than CC carriers (log rank test, $P=0.001$ ), while patients carrying TC genotype had obviously better PFS than those carrying CC genotype (log rank test, $P=0.013)$. There was no remarkable difference between TT carriers and TC carriers ( $\log$ rank test, $P=0.126)$. The significant level was adjusted by Bonferroni method, $\alpha=0.017$. 
Table 3: The effects of VEGFA rs833061 polymorphism on ORR, DCR and PFS among CRC patients receiving bevacizumab treatment

\begin{tabular}{|c|c|c|c|c|c|c|c|c|c|c|c|c|}
\hline \multirow{2}{*}{ Genotypes } & \multicolumn{4}{|c|}{ ORR } & \multicolumn{4}{|c|}{ DCR } & \multicolumn{4}{|c|}{ PFS } \\
\hline & OR & $P$ & $\mathrm{OR}^{\mathrm{a}}$ & $P^{a}$ & OR & $P$ & $\mathrm{OR}^{\mathrm{a}}$ & $P^{a}$ & HR & $P$ & $H_{R^{b}}$ & $P^{b}$ \\
\hline TT & $\begin{array}{c}0.220 \\
(0.044-1.103)\end{array}$ & 0.048 & $\begin{array}{c}0.224 \\
(0.044-1.134)\end{array}$ & 0.071 & $\begin{array}{c}0.129 \\
(0.031-0.532)\end{array}$ & 0.002 & $\begin{array}{c}0.124 \\
(0.030-0.519)\end{array}$ & 0.004 & $\begin{array}{c}0.299 \\
(90.141-0.634)\end{array}$ & 0.002 & $\begin{array}{c}0.299 \\
(0.141-0.634)\end{array}$ & 0.002 \\
\hline $\mathrm{TC}$ & $\begin{array}{c}0.159 \\
(0.029-0.861)\end{array}$ & 0.021 & $\begin{array}{c}0.159 \\
(0.029-0.871)\end{array}$ & 0.034 & $\begin{array}{c}0.241 \\
(0.054-1.070)\end{array}$ & 0.052 & $\begin{array}{c}0.230 \\
(0.051-1.038)\end{array}$ & 0.056 & $\begin{array}{c}0.449 \\
(0.203-0.991)\end{array}$ & 0.048 & $\begin{array}{c}0.449 \\
(0.203-0.991)\end{array}$ & 0.048 \\
\hline $\mathrm{CC}$ & \multicolumn{4}{|c|}{ Reference } & \multicolumn{4}{|c|}{ Reference } & \multicolumn{4}{|c|}{ Reference } \\
\hline
\end{tabular}

Notes: a: calculated by logistic regression analysis; b: analyzed by cox-regression analysis.

response rate [28]. Loupakis et al. suggested that rs699947 variant could not impact the PFS and OS in CRC patients in Caucasian population [29]. Schneider et al. suggested that rs833061 TT might decrease the hypertension toxicity of BEV [30]. VEGF -2578CC and -1498TT genotypes decreased the hypertension toxicity of BEV in Japanese CRC patients [31], but these two SNPs had no association with the outcome of CRC patients treated with BEV-based chemotherapy [32]. However, other studies were different from our results. For instance, Loupakis et al. revealed that rs833061 TT genotype associated with shorter PFS in Italian CRC patients who received first-line FOLFIRI plus BEV treatment [29]. The divergence may be caused by the diversity of regions, races, select criteria or the categories of disease.

In conclusion, VEGFA rs833061 polymorphism is obviously associated with the therapeutic efficiency of BEV in Chinese Han CRC patients. This result will help us to select the patients who exert high response to BEV treatment, thus improving the clinical outcomes of CRC patients. However, our results may be limited by the relatively small sample size and the single ethnicity in the current study. Additionally, the concrete molecular mechanisms underlying the genetic association of VEGFA rs833061 polymorphism with BEV treatment are not investigated in the current study. Further investigations will be required to verify the conclusion.

\section{MATERIALS AND METHODS}

\section{Study subjects}

This study was approved by the local ethics committee. All of the participants were unrelated Chinese Han people. They understood the objectives of the study and signed the informed consents. The sample collection was in accordance with the Helsinki declaration.

Study subjects were selected from CRC patients who were hospitalized in The Military General Hospital of Beijing PLA from January 2010 to January 2013. Patients were diagnosed with CRC by computed tomography (CT) and histopathologic examinations based on the diagnosis standards of CRC [33]. Include criteria were as follows: CRC patients who had metastasis or postoperative recurrence were included in this study. Patients had the following conditions were excluded from this study: expectancy life was less than 3 months; received antiangiogenic therapy before this study; and had any other cardiovascular or cerebrovascular diseases. Patients who had the adjuvant treatment less than 6 cycles were also excluded from this study. Finally, 125 patients (age range 31-80 years) were recruited in this study.

\section{Therapy methods and therapeutic efficacy}

All the patients received $\mathrm{BEV}(5 \mathrm{mg} / \mathrm{kg}$, every 2 weeks) plus FOLFIRI treatment. FOLFIRI therapeutic regimen included Irinotecan $180 \mathrm{mg} / \mathrm{m}^{2}$, 1-leucovorin $200 \mathrm{mg} / \mathrm{m}^{2}$, and 5-fluorouracil $400 \mathrm{mg} / \mathrm{m}^{2}$, intravenous injection on day 1 , followed by a 4-6hrs infusion of 5-fluorouracil 2,400 mg/m², every 2 weeks.

Treatment response was evaluated by experienced oncologists according to RECIST 1.1 after three months treatment [34]. Treatment response included complete response (CR), partial response (PR), stable disease (SD) and progressive disease (PD). The short therapeutic efficiency was estimated by object response rate (ORR) and disease control rate (DCR). The rate of $\mathrm{CR}+\mathrm{PR}$ is defined as ORR. DCR is defined as the rate of $\mathrm{CR}+\mathrm{PR}+\mathrm{SD}$. The long effects of VEGFA variants on BEV treatments were evaluated by progression-free survival (PFS). PFS is the duration from the beginning of the treatment to the first observation of disease progression or the last follow-up visit. Follow up time was 30 months. Patients were diagnosed by CT scan every 3 months.

\section{Genotyping analysis}

$5 \mathrm{ml}$ peripheral venous blood was collected form each participant who had a $12 \mathrm{~h}$ fasting before the adjuvant treatment. Blood samples were anti-coagulated by EDTA, and stored at $-80^{\circ} \mathrm{C}$ until used. Genomic DNA was extracted by a TIANamp Blood DNA Kit (Tiangen, China), following the manufacturer's protocol.

SNPs of $V E G F$ gene were analyzed by polymerase chain reaction-restriction fragment length polymorphism (PCR-RFLP), according to the previous study [35]. Genotype results were confirmed by direct sequencing 
method using ABI 3730XL sequencer via randomly selected 50 samples.

\section{Statistical analysis}

All of the statistic analyses were conducted by PASW 18.0 software. Significant level was set to two side 0.05 and adjusted by Bonferroni method in multiple comparison. Genotype and allele distributions were detected by Hardy-Weinberg equilibrium (HWE) test. Genotype and allele frequencies were obtained by direct counting. Quantitative variables were shown as mean \pm standard deviation. Qualitative variables were analyzed by Chi-square test. CR, PR, SD and PD were used to present the responses for bevacizumab treatment. Kaplan-Meier survival curve was utilized to analyze the PFS of the patients after bevacizumab therapy. Timeto-event distributions were analyzed by log-rank test, and the results were adjusted using Bonferroni method. Multivariate logistic regression analysis and multivariate cox regression analysis were performed to estimate the association of $V E G F$ polymorphisms on bevacizumab efficiency after adjusting to the other confusing factors.

\section{CONFLICTS OF INTEREST}

The authors declare no conflicts of interest.

\section{REFERENCES}

1. Siegel R, Desantis C, Jemal A. Colorectal cancer statistics, 2014. CA Cancer J Clin. 2014; 64:104-117.

2. Jiang K, Sun Y, Wang C, Ji J, Li Y, Ye Y, Lv L, Guo Y, Guo S, Li H, Zhang L, Zhou Y, Jiang B, et al. Genomewide association study identifies two new susceptibility loci for colorectal cancer at 5q23.3 and 17q12 in Han Chinese. Oncotarget. 2015; 6:40327-40336. https://doi.org/10.18632/ oncotarget.5530.

3. Song M, Mehta RS, Wu K, Fuchs CS, Ogino S, Giovannucci EL, Chan AT. Plasma inflammatory markers and risk of advanced colorectal adenoma in women. Cancer Prev Res (Phila). 2016; 9:27-34.

4. Anderson AS, Caswell S, Macleod M, Craigie AM, Stead M, Steele RJ, Team TB. Awareness of lifestyle and colorectal cancer risk: findings from the BeWEL study. Biomed Res Int. 2015; 2015:871613.

5. Jung YS, Yun KE, Chang Y, Ryu S, Park DI. Risk factors such as male sex, smoking, metabolic syndrome, obesity, and fatty liver do not justify screening colonoscopies before age 45. Digest Dis Sci. 2016; 61:1021-1027.

6. Zhao Y, Adjei AA. Targeting angiogenesis in cancer therapy: moving beyond vascular endothelial growth factor. Oncologist. 2015; 20:660-673.

7. Simons M, Eichmann A. Molecular controls of arterial morphogenesis. Circ Res. 2015; 116:1712-1724.
8. Cydzik M, Abdul-Wahid A, Park S, Bourdeau A, Bowden K, Prodeus A, Kollara A, Brown TJ, Ringuette MJ, Gariepy J. Slow binding kinetics of secreted protein, acidic, rich in cysteine-VEGF interaction limit VEGF activation of VEGF receptor 2 and attenuate angiogenesis. FASEB J. 2015; 29:3493-3505.

9. Oh HH, Park KJ, Kim N, Park SY, Park YL, Oak CY, Myung DS, Cho SB, Lee WS, Kim KK, Joo YE. Impact of KITENIN on tumor angiogenesis and lymphangiogenesis in colorectal cancer. Oncol Rep. 2016; 35:253-260.

10. Heath VL, Bicknell R. Anticancer strategies involving the vasculature. Nat Rev Clin Oncol. 2009; 6:395-404.

11. Zhao YJ, Han HZ, Liang Y, Shi CZ, Zhu QC, Yang J. Alternative splicing of VEGFA, APP and NUMB genes in colorectal cancer. World J Gastroenterol. 2015; 21:6550-6560.

12. Langmia IM, Apalasamy YD, Omar SZ, Mohamed Z. Association of VEGFA gene polymorphisms and VEGFA plasma levels with spontaneous preterm birth. Pharmacogenet Genomics. 2015; 25:199-204.

13. Wu X, Xin Z, Zhang W, Wu J, Chen K, Wang H, Zhu X, Pan L, Li Z, Li H, Liu Y. Polymorphisms in the VEGFA promoter are associated with susceptibility to hepatocellular carcinoma by altering promoter activity. Int J Cancer. 2013; 133:1085-1093.

14. Balasubramanian SP, Brown NJ, Reed MW. Role of genetic polymorphisms in tumour angiogenesis. Br J Cancer. 2002; 87:1057-1065.

15. Credidio L, Lima CS, Leal R, de Ayrizono ML, Fagundes JJ, Magna LA, Coy CS. C936T polymorphism of the VEGF gene in relation to the risk and the clinical and biological characteristics of sporadic colorectal adenocarcinoma. BMC Res Notes. 2014; 7:768.

16. Kapahi R, Guleria K, Sambyal V, Manjari M, Sudan M, Uppal MS, Singh NR. Association of VEGF and VEGFR1 polymorphisms with breast cancer risk in North Indians. Tumour Biol. 2015; 36:4223-4234.

17. Yang PW, Hsieh MS, Huang YC, Hsieh CY, Chiang TH, Lee JM. Genetic variants of EGF and VEGF predict prognosis of patients with advanced esophageal squamous cell carcinoma. PLoS One. 2014; 9:e100326.

18. Ferroni P, Palmirotta R, Riondino S, De Marchis ML, Nardecchia A, Formica V, Guadagni F, Roselli M. VEGF gene promoter polymorphisms and risk of VTE in chemotherapy-treated cancer patients. Thromb Haemost. 2016; 115:143-151.

19. Allegrini G, Coltelli L, Orlandi P, Fontana A, Camerini A, Ferro A, Cazzaniga M, Casadei V, Lucchesi S, Bona E, Di Lieto M, Pazzagli I, Villa F, et al. Pharmacogenetic interaction analysis of VEGFR-2 and IL-8 polymorphisms in advanced breast cancer patients treated with paclitaxel and bevacizumab. Pharmacogenomics. 2014; 15:1985-1999.

20. Di Stefano AL, Labussiere M, Lombardi G, Eoli M, Bianchessi D, Pasqualetti F, Farina P, Cuzzubbo S, 
Gallego-Perez-Larraya J, Boisselier B, Ducray F, Cheneau C, Moglia A, et al. VEGFA SNP rs2010963 is associated with vascular toxicity in recurrent glioblastomas and longer response to bevacizumab. J Neurooncol. 2015; 121:499-504.

21. Sohn BS, Park SJ, Kim JE, Kim KP, Hong YS, Suh C, Kim YS, Kim SY, Im SA, Kim JH, Ahn JB, Park YS, Kim TW. Single-nucleotide polymorphisms in the vascular endothelial growth factor pathway and outcomes of patients treated with first-line cytotoxic chemotherapy combined with bevacizumab for advanced colorectal cancer. Oncology. 2014; 87:280-292.

22. Hein A, Lambrechts D, von Minckwitz G, Haberle L, Eidtmann H, Tesch H, Untch M, Hilfrich J, Schem C, Rezai M, Gerber B, Dan Costa S, Blohmer JU, et al. Genetic variants in VEGF pathway genes in neoadjuvant breast cancer patients receiving bevacizumab: Results from the randomized phase III GeparQuinto study. Int J Cancer. 2015; 137:2981-2988.

23. Baluk P, Hashizume H, McDonald DM. Cellular abnormalities of blood vessels as targets in cancer. Curr Opin Genet Dev. 2005; 15:102-111.

24. Johnsson A, Hagman H, Frodin JE, Berglund A, Keldsen N, Fernebro E, Sundberg J, De Pont Christensen R, Garm Spindler KL, Bergstrom D, Jakobsen A. A randomized phase III trial on maintenance treatment with bevacizumab alone or in combination with erlotinib after chemotherapy and bevacizumab in metastatic colorectal cancer: the Nordic ACT Trial. Ann Oncol. 2013; 24:2335-2341.

25. Grapsa D, Syrigos K, Saif MW. Bevacizumab in combination with fluoropyrimidine-irinotecan- or fluoropyrimidine-oxaliplatin-based chemotherapy for firstline and maintenance treatment of metastatic colorectal cancer. Expert Rev Anticancer Ther. 2015; 15:1267-1281.

26. Bayhan Z, Simsek T, Ergul E, Utkan NZ, Canturk NZ, Cekmen M. Serum cytokine levels in patients with colorectal cancers according to tumor stages and VEGF gene polymorphism. Hepatogastroenterology. 2014; 61:1889-1894.

27. Stremitzer S, Zhang W, Yang D, Ning Y, Sunakawa Y, Matsusaka S, Parekh A, Okazaki S, Hanna D, Astrow SH, Moran M, Hernandez J, Stephens C, et al. Expression of genes involved in vascular morphogenesis and maturation predicts efficacy of bevacizumab-based chemotherapy in patients undergoing liver resection. Mol Cancer Therapeut. 2016; 15:2814-2821.
28. Pallaud C, Reck M, Juhasz E, Szima B, Yu CJ, Burdaeva O, Orlov S, Hilton M, Archer V, Mok T. Clinical genotyping and efficacy outcomes: exploratory biomarker data from the phase II ABIGAIL study of first-line bevacizumab plus chemotherapy in non-squamous non-small-cell lung cancer. Lung Cancer. 2014; 86:67-72.

29. Loupakis F, Cremolini C, Yang D, Salvatore L, Zhang W, Wakatsuki T, Bohanes P, Schirripa M, Benhaim L, Lonardi $\mathrm{S}$, Antoniotti C, Aprile G, Graziano F, et al. Prospective validation of candidate SNPs of VEGF/VEGFR pathway in metastatic colorectal cancer patients treated with first-line FOLFIRI plus bevacizumab. PLoS One. 2013; 8:e66774.

30. Schneider BP, Radovich M, Miller KD. The role of vascular endothelial growth factor genetic variability in cancer. Clin Cancer Res. 2009; 15:5297-5302.

31. Morita S, Uehara K, Nakayama G, Shibata T, Oguri T, Inada-Inoue M, Shimokata T, Sugishita M, Mitsuma A, Ando Y. Association between bevacizumab-related hypertension and vascular endothelial growth factor (VEGF) gene polymorphisms in Japanese patients with metastatic colorectal cancer. Cancer Chemother Pharmacol. 2013; 71:405-411.

32. Koutras AK, Antonacopoulou AG, Eleftheraki AG, Dimitrakopoulos FI, Koumarianou A, Varthalitis I, Fostira F, Sgouros J, Briasoulis E, Bournakis E, Bafaloukos D, Bompolaki I, Galani E, et al. Vascular endothelial growth factor polymorphisms and clinical outcome in colorectal cancer patients treated with irinotecan-based chemotherapy and bevacizumab. Pharmacogenomics J. 2012; 12:468-475.

33. Dominic OG, McGarrity T, Dignan M, Lengerich EJ. American College of Gastroenterology Guidelines for colorectal cancer screening 2008. Am J Gastroenterol. 2009; 104:2626-2627; author reply 2628-2629.

34. Eisenhauer EA, Therasse P, Bogaerts J, Schwartz LH, Sargent D, Ford R, Dancey J, Arbuck S, Gwyther S, Mooney M, Rubinstein L, Shankar L, Dodd L, et al. New response evaluation criteria in solid tumours: revised RECIST guideline (version 1.1). Eur J Cancer. 2009; 45:228-247.

35. Loupakis F, Ruzzo A, Salvatore L, Cremolini C, Masi G, Frumento P, Schirripa M, Catalano V, Galluccio N, Canestrari E, Vincenzi B, Santini D, Bencardino K, et al. Retrospective exploratory analysis of VEGF polymorphisms in the prediction of benefit from first-line FOLFIRI plus bevacizumab in metastatic colorectal cancer. BMC Cancer. 2011; 11:247. 